\title{
A COMPUtATIONAL MOdel FOR MULTI-CRITERIA DeCISION MAKING In TRAFFic Jam Problem
}

\section{Ali Naeem, Jabbar Abbas}

\section{DOI: 10.14313/JAMRIS/2-2021/12}

\begin{abstract}
In this paper, we apply a computational model for multicriteria decision making in traffic jam problems. First, we propose a system to determine the optimal shortcut road by reading the number of cars in each street using $R a-$ dio Frequency Identification (RFID). Then, we have processed the data of traffic jam problems using Choquet integral with writing algorithm and computer program as a working procedure.
\end{abstract}

Keywords: Multi-Criteria Decision Making, Fuzzy Measures, Choquet Integrals, Traffic Jams

\section{Introduction}

Traffic jam is the worst problem that drivers are suffering in their daily lives, both in terms of delays in the traffic jams when going to work or the inconvenience caused by road jam in cars on public holidays. The problem of traffic congestion is not confined to regions without other regions, and thus countries always seek to address it in all kinds of solutions. The theory of multi-criteria decision-making has gained the emergence of several new paradigms in the second half of the twentieth century (for more details see e.g. [1]). There are several multi-criteria decisionmaking approaches that have been introduced to study the problem of traffic congestion (see e.g. [2]).

The aim of this paper is to apply a computational model for multi-criteria decision making in traffic jam problems, then use a system as a working procedure, to determine the optimal shortcut road using Radio Frequency Identification (RFID). The RFID system has already been adopted into various application areas (see, [3]-[6]).

In this paper, we propose a system that processed the data of traffic jam problems using Choquet integral to give the best result of the most appropriate road (optimal shortcut road) to go from the start point to the endpoint.

The structure of this paper is as follows. Section 2 introduces Multi Criteria Decision Making problem. In section 3, we propose a system to determine the optimal shortcut road in traffic jams. In section 4 , we give a practical example. In section 5 , we finish the paper with some conclusions.

\section{Multi-Criteria Decision Making Problem}

Consider a multi-criteria decision making problem that depends on $n$ criteria (or points of view, attribute) described by the alternatives

$G_{1}, \ldots, G_{n}$ and a set of criteria $\mathrm{X}=\{1, \ldots, n\}$. An alternative is characterized by a value with respect to each criterion and is thus identified with a point in the Cartesian product $G$ of the criteria, i.e. $G=G_{1} \times \ldots \times G_{n}$ is the set of potential alternatives.

The preference relation of the Decision Maker (DM) over alternatives is denoted by $\geq$. For any $g, h \in G, g \geq h$ means that the DM prefers alternative $g$ to $h$. A classical way is to model $\geq$ with the help of an overall utility function $z: G \rightarrow R$ [1]:

$\forall g, h \in \mathrm{G}, \quad g \geq h \leftrightarrow z(g) \geq z(h)$.

Clearly, $z$ is a $n$ dimensional function. An easy way to construct $z$ is to consider one-dimensional utility function $z_{i}$ on each criterion and then to aggregate them by a suitable operator:

$z(g)=F\left(z_{1}\left(g_{1}\right), \cdots, Z_{n}\left(g_{n}\right)\right) \quad \forall g \in G$

where, $\mathrm{F}$ is called an aggregation function.

Aggregation functions (AFs) are mathematical functions to collect helpful data in multi criteria decision making. The input of AFs is several numerical values and its output is a single value.

Based on the overall score by means of an aggregation function that takes into account the weights of importance of the criteria, the alternatives can be ranked and the best alternative selected. A special type of aggregation function is fuzzy integral with respect to non-additive measure. The fuzzy integral [7], [8] is an appropriate tool to represents the weights of criteria with non-additive measures. One of the fuzzy integrals is Choquet integral with respect to non-additive measure (fuzzy measure). The fuzzy integrals with respect to non-additive measures have been studied and applied in diverse fields (see, e.g. [9-17]). There are many types of non-additive measures, one of them is the $\lambda$-fuzzy measure. The definition of $\lambda$-fuzzy measure is as follows.

Definition 1: Suppose $P(G)$ be the power set of $G$, $A$ set function is called $\lambda$-fuzzy measure if it satisfies the following axioms:

1. $g_{\lambda}: P(G) \rightarrow[0,1]$

2. If $S, T \in P(G), S \cap T=\varnothing$, then 


$$
\begin{gathered}
g_{,}(S \cup \mathrm{T})=g_{,}(S)+g_{,}(\mathrm{T})+2 g_{,}(S) g_{,}(\mathrm{T}), \\
\lambda \in(-1, \infty)
\end{gathered}
$$

In general, it can be shown that

$$
g_{2}\left(\left\{x_{1}, \ldots, x_{n}\right\}\right)=1=\frac{1}{2}\left[\prod_{i=1}^{n}\left(1+2 g_{2}\left(x_{i}\right)\right)-1\right], \mathrm{\lambda}=0
$$

This given a polynomial equation with respect to $\lambda$

$$
1+\mathrm{z}=\prod_{i=1}^{n}\left(1+\mathrm{z} g,\left(x_{i}\right)\right) \text {. }
$$

The mapping $f: G \rightarrow R+$ corresponds to the value that the supply of the source (i.e., $f\left(x_{\mathrm{i}}\right)=x_{\mathrm{i}}$ ), using, as before, $x_{\mathrm{i}}$ to denote the $i_{\text {th }}$ input value and the fuzzy measure $\left(g_{\lambda}: P(G) \rightarrow[0,1]\right)$ assigns importance to subsets of $G$.

Definition 2: Suppose that $g_{\lambda}$ is fuzzy measures on $G$ whose elements are, refer to $\left(x_{1}, \ldots, x_{\mathrm{n}}\right)$. The discrete choquet integral of a function $f: G \rightarrow R+$ with respect to $g_{\lambda}$ is defined by

$$
\int C_{g_{2}}(f)=\sum_{i=1}^{N}\left(f\left(x_{(i)}\right)-f\left(x_{(i-1)}\right)\right) g,\left(A_{(i)}\right)
$$

where (i) denotes that the indices have been permuted so that

$$
0 \leq f\left(X_{(1)}\right) \leq \cdots \leq f\left(X_{(n)}\right) \text {, and } A_{(i)}:=\left\{X_{(i)}, \cdots, X_{(\mathrm{n})}\right\}
$$

with $f\left(x_{(0)}\right)=0$

\section{A Computational Model for Multi-Criteria Decision Making in Traffic Jam Problem} 3.1 A System for Solving the Problem of Traffic Jams

In order to solve the problem of traffic jams, we must first try to understand its causes. While the causes and may differ from city to city, there are some common factors. For example, the increase in the number of cars and narrow roads are obvious reasons. Hence the idea of the solution of traffic jams through which the street jam is identified by knowing the number of cars in each street of the city that the user wants to pass. This process is done using the Radio Frequency Identification (RFID) technique, through which scores every car entering and go out the specified road.

The procedure of statistical work for the proposed roads and choose the most appropriate road among these roads through the processing of data using multi-criteria decision making problems. Thus, the user can query the best road through an application designed for this purpose that is available for all smart mobile devices. Some countries use GPS technology to define the number of cars and regardless of the economic cost of this technology, where the cost of GPS is more than the cost of RFID. Furthermore, it is not available in all cars and it's difficult for the normal driver to install it comparing with the RFID technology used in our system.

\subsection{Hardware Design of the System}

The Hardware design of the system can be classified into the following.

Arduino. An electronic development board (as in Figure 1), can be used by both professionals and beginners, and it is used in different fields. Arduino can communicate with the surrounding environment through a number of sensors and can influence its surroundings by controlling small motors or lights and other electronic parts. Arduino's projects can be connected to its sensors and electronic parts only or can be connected to computer-based programs, such as Visual BASIC.

Radio Frequency Identification (RFID). RFID is one of the most common applications in the recent period (Figure 2). It is widely found in the security system and is done by sending electromagnetic waves to special cards (Figure 3).

The hardware design of the system for solving the problem of traffic jams is shown in the following figures (Figure 4 and Figure 5).

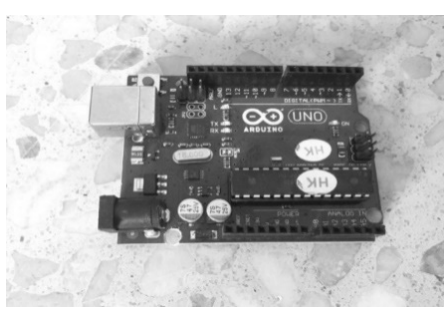

Fig. 1. Arduino Uno board

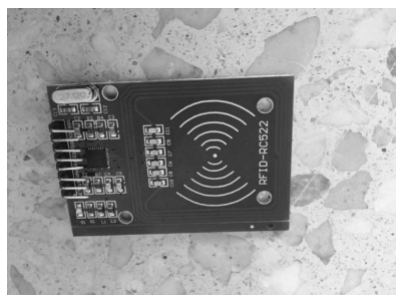

Fig. 2. RFID board

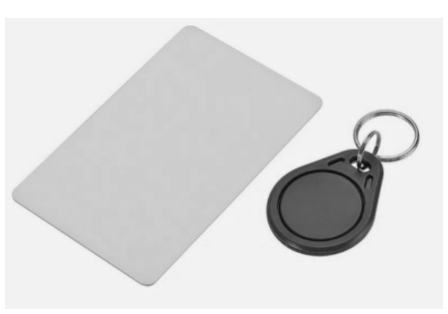

Fig. 3. Tags (RF Sender)

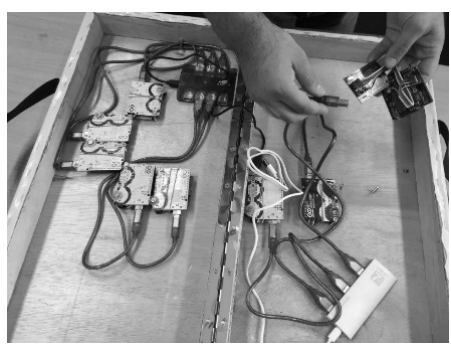

Fig. 4. Hardware for a small model 


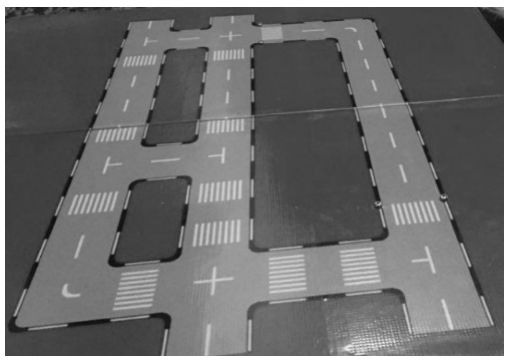

Fig. 5. A small city map

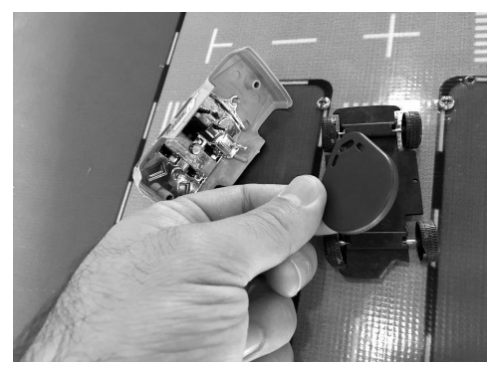

Fig. 6. A small car with tag inside

In the practical application will get the values of three criteria (Length, Time, and Traffic jam) automatically through the RFID reader (Figure 6, Figure 7) which will calculate the number of cars on the road, and calculate the rate of cars on the road by dividing the number of cars on the street capacity, and this value will represent the road jam. When the user wants to choose the best road, the application will draw the best streets on the map (Figure 10). The length of the street will be taken from the Google maps application, and the time will depend on the street length and the traffic jam. The hardware design of the system tools is showing in the following figures (Figure 6- Figure 9).

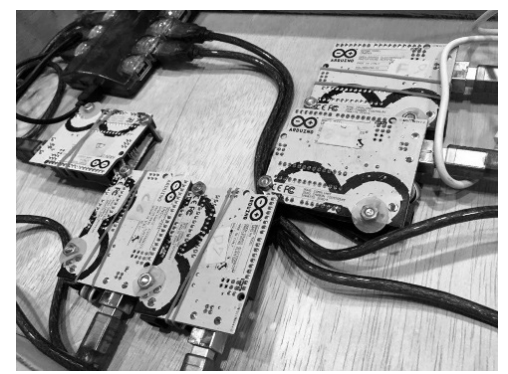

Fig. 7. Arduino connection

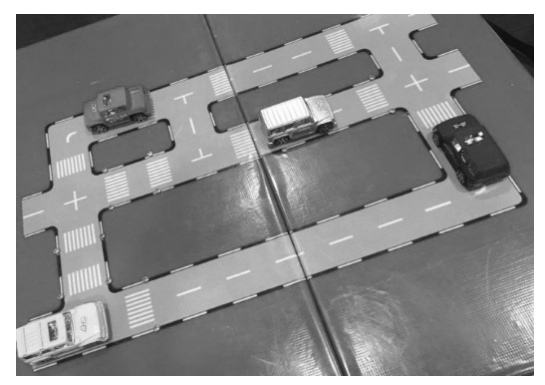

Fig. 8. Small city simulator

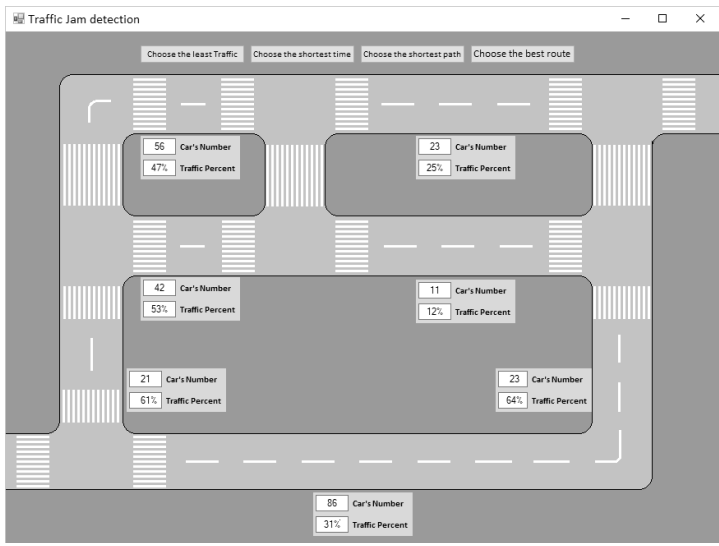

Fig. 9. Before choosing a path

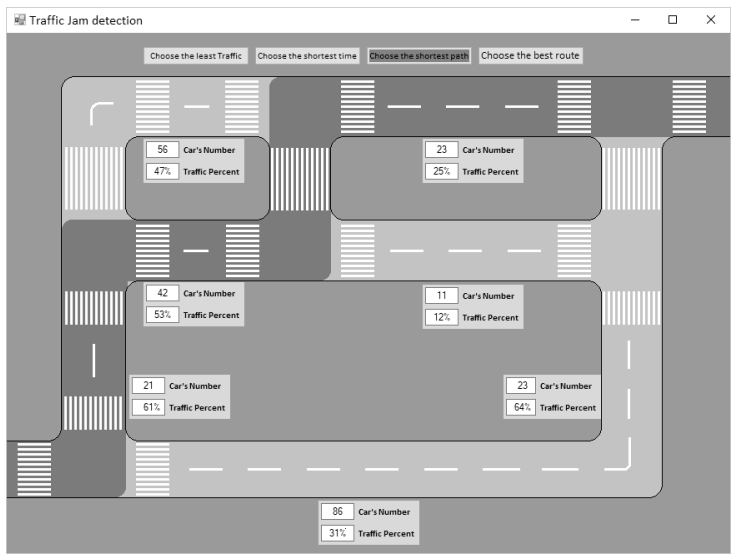

Fig. 10. The shortest path

\subsection{Algorithm}

To simplify the notation in the algorithm, we use the following equation to calculate the discrete Choquet integral that is equivalent to the equation (6):

$$
\int C_{g_{3}}(f)=\sum_{i=1}^{n}\left[x_{(i)}-x_{(i-1)}\right] g_{,}\left(A_{(i)}\right)
$$

where $x_{(0)}=0$ by convention, and $A_{(\mathrm{i})}=\{1, \ldots, n\}$

Consider the vector of pairs $\left(\left(x_{1}, 1\right),\left(x_{2}, 2\right), \ldots\right.$, $\left.\left(x_{\mathrm{n}}, n\right)\right)$, where the second component of each pair is just the index $i$ of $x_{\mathrm{i}}$. The second component will help to keep track of all permutations. Therefore, we use the following procedure for the calculation of the discrete Choquet integral $C_{g}(f)$.

Step 1: Sort the components of $\left(\left(x_{1}, 1\right),\left(x_{2}, 2\right), \ldots\right.$, $\left.\left(x_{\mathrm{n}}, n\right)\right)$ with respect to the first component of each pair in non-decreasing order.

We obtain,

$\left(\left(x_{(1)}, i_{1}\right),\left(x_{(2)}, i_{2}\right), \ldots,\left(x_{(\mathrm{n})}, i_{\mathrm{n}}\right)\right)$, so that $x_{(\mathrm{j})}=x i_{\mathrm{j}}$ and $x_{(j)} \leq x_{(\mathrm{j}+1)}$ for all $i$.

Let also $x_{(0)}=0$.

Step 2: Let $T=\{1, \ldots, n\}$, and $S=0$.

Step 3: For $j=1, \ldots, n$ do

a) $S:=S+\left[x_{(\mathrm{j})}-x_{(\mathrm{j}-1)}\right] g_{\lambda}(T)$;

b) $T:=T \mid\left\{i_{\mathrm{j}}\right\}$

Step 4: Return $S$. 


\section{Practical Example}

We consider the problem of evaluation of traffic jams on roads with respect to three criteria, Length (L), Time (T), and Traffic Jam (J). This is usually done through a simple weighted amount, in which weights are coefficients of the importance of different criteria. Let us take some areas on the map that contain five roads ( $R 1, R 2, R 3, R 4, R 5)$ to move from point (A) to point (B). The data of the five roads are given in the following table (Table 1).

Tab. 1. The data of the five roads ( $R 1, R 2, R 3, R 4, R 5)$

\begin{tabular}{|c|c|c|c|}
\hline & Length (L) & Time (T) & Traffic Jam(J) \\
\hline R1 & 200 & 100 & 55 \\
\hline R2 & 300 & 180 & 55 \\
\hline R3 & 150 & 70 & 90 \\
\hline R4 & 150 & 60 & 80 \\
\hline R5 & 150 & 50 & 70 \\
\hline
\end{tabular}

We can solve this problem by using a suitable fuzzy measure and the Choquet integral as follows.

1. The Length and Traffic jam criteria are more important than Time, we put the following weights on criteria taken individually.

$g,(\{$ Length $\})=0.7, g,(\{$ Time $\})=0.5, g,(\{$ Traffic Jam $\})=0.6$.

Using equation (5), we get

$$
1+\lambda=(1+0.7 x)(1+0.5 x)(1+0.6 a)
$$

Then, the solutions of this equation are $\lambda=-0.9103$, $\lambda=-4.1849$, and $\lambda=0$. The only acceptable value is $\lambda=-0.9103$, but the other values are violated of constraints of $\lambda$-fuzzy measure.

2. Since length and traffic jam are redundant, the weight attributed to the length and the traffic jam should be less than the total weights of the length and traffic jams. By applying equation (3), $g,\left(\left\{x_{1}, x_{3}\right\}\right)=$,

$g_{,}\left(\left\{x_{1}\right\}\right)+g_{2}\left(\left\{x_{3}\right\}\right)+2 g,\left(\left\{x_{1}\right\}\right) g_{2}\left(\left\{x_{3}\right\}\right)$

$g,\left(\left\{x_{1}, x_{3}\right\}\right)=g,(\{$ length, traffic jam $\})$

$=0.8+0.5+(-0.9439 * 0.8 * 0.5)=0.917674$

$g,(\{$ length, traffic jam $\})=0.917674<0.8+0.5$.

Similarly, the weight attributed to the time and length should be greater than the sum of individual weights (also, the same for time and traffic jam).

$g,\left(\left\{x_{1}, x_{2}\right\}\right)=g,(\{$ length, time $\})=0.881395<0.8+0.6$,

$g,\left(\left\{x_{2}, x_{3}\right\}\right)=g,(\{$ time, traffic jam $\})=0.82691<0.6+0.5$,

$g,(\varnothing)=0, g,(\{$ length, traffic jam, time $\})=1$.
3. By applying $\lambda$-fuzzy measure and Choquet integral for the first road (R1) we get the following result for (R1). $0 \leq f\left(x_{3}\right) \leq f\left(x_{2}\right) \leq f\left(x_{1}\right)$,

$$
\begin{aligned}
\int C_{g_{2}}(f)= & \sum_{i=1}^{N}\left(f\left(x_{(i)}\right)-f\left(x_{(i-1)}\right)\right) g_{2}\left(A_{(i)}\right) \\
\int C_{g_{2}}(R 1) & =\left[f\left(x_{3}\right)-f(0)\right] g_{2}\left(x_{1}, x_{2}, x_{3}\right) \\
& +\left[f\left(x_{2}\right)-f\left(x_{3}\right)\right] g_{2}\left(x_{1}, x_{2}\right) \\
& +\left[f\left(x_{1}\right)-f\left(x_{2}\right)\right] g_{2}\left(x_{1}\right)
\end{aligned}
$$

$$
\begin{aligned}
\int C_{g_{2}}(R 1) & =[55-0] * 1+[100-55] * 0.88139 \\
& +[200-100] * 0.7 \\
& =164.66
\end{aligned}
$$

Other results for the remaining roads (R2, R3, R4, R5) are shown in Table 2.

Tab. 2. Choquet integral for the roads $(R 1, R 2, R 3, R 4, R 5)$

\begin{tabular}{|c|c|c|c|c|}
\hline & Length (L) & Time(T) & Jam (J) & $\begin{array}{c}\text { Global evaluation } \\
\text { (Choquet integral) }\end{array}$ \\
\hline Road 1 & 200 & 100 & 55 & 164.66 \\
\hline Road 2 & 300 & 180 & 55 & 249.17 \\
\hline Road 3 & 150 & 50 & 70 & 124.35 \\
\hline Road 4 & 150 & 60 & 80 & 127.35 \\
\hline Road 5 & 150 & 70 & 90 & 130.35 \\
\hline
\end{tabular}

The interpretation of Choquet integral with respect to scoring (from lower to above) on the road ranking confirms that the preference relation of the Decision Maker is $\mathrm{R} 3 \succeq \mathrm{R} 4 \succeq \mathrm{R} 5 \succeq \mathrm{R} 1 \succeq \mathrm{R} 2$.

The refore, Road 5 is the optimal shortcut road according to the ranking roads. Thus, we get the predictable solution of the traffic jam problem for the appropriate road (optimal shortcut path) to go from the start point to the endpoint.

\section{Conclusion}

In this paper, we have described a computational model of the problem of multi-criteria decision making in traffic jams. Then, we have proposed in this model a system for solving the Multi-Criteria Decision Making Problem of traffic jams. The application of this system is high speed in data collection and processing, also the mechanism of the system is automatic. In the future, we plan to test our model in many areas, such as service, commercial, and security fields.

\section{AUTHORS}

Ali Naeem - Department of Applied Sciences, University of Technology, Baghdad, Iraq, Email: aliallamy. itu@gmail.com.

Jabbar Abbas* - Department of Applied Sciences, University of Technology, Baghdad, Iraq, Email: 100033@uotechnology.edu.iq. 


\section{REFERENCES}

[1] J. Figueira, S. Greco and M. Ehrogott, Multiple Criteria Decision Analysis: State of the Art Surveys, Springer New York, 2005, 10.1007/b100605.

[2] N. Hao, Y. Feng, K. Zhang, G. Tian, L. Zhang and $H$. Jia, "Evaluation of traffic congestion degree: An integrated approach", Int. J. Distrib. Sens. Netw., vol. 13, no. 7, 2017, $10.1177 / 1550147717723163$.

[3] D. Yue, X. Wu and J. Bai, "RFID Application Framework for pharmaceutical supply chain". In: 2008 IEEE International Conference on Service Operations and Logistics, and Informatics, 2008, 1125-1130, 10.1109/SOLI.2008.4686568.

[4] A. Hamdan, M. Chen and K. J. Rogers, "RFID application in the third-party logistics industry". In: 2006 Technology Management for the Global Future - PICMET 2006 Conference, 2006, 27692795, 10.1109/PICMET.2006.296871.

[5] D. Manik, L. Toth and P. Dobrossy, "Analysis of RFId Application Through an Automotive Suppliers Production Processes". In: 2007 International Symposium on Computational Intelligence and Intelligent Informatics, 2007, 177-181, 10.1109/ISCIII.2007.367385.

[6] S.-W. Wang, W.-H. Chen, C.-S. Ong, L. Liu and Y.-W. Chuang, "RFID Application in Hospitals: A Case Study on a Demonstration RFID Project in a Taiwan Hospital". In: Proc. of the 39th Annual Hawaii International Conference on System Sciences (HICSS'06), 2006, 10.1109/HICSS.2006.422.

[7] G. Choquet, "Theory of capacities", Ann. Inst. Fourier, vol. 5, 1954, 131-295, 10.5802/aif.53.

[8] M. Sugeno, "Theory of fuzzy integrals and its applications", Ph. D. dissertation, Tokyo Institute of Technology, Tokyo, 1974.

[9] A. Mendez-Vazquez, P. Gader, J. M. Keller and K. Chamberlin, "Minimum Classification Error Training for Choquet Integrals With Applications to Landmine Detection", IEEE Trans. Fuzzy Syst., vol. 16, no. 1, 2008, 225-238, 10.1109/ TFUZZ.2007.902024.

[10] J. Abbas, "The Bipolar Choquet Integrals Based on Ternary-Element Sets", Journal of Artificial Intelligence and Soft Computing Research, vol. 6, no. 1, 2016, 13-21, 10.1515/jaiscr-2016-0002.

[11] J. Abbas, "The Balancing Bipolar Choquet Integrals", Int. J. Innov. Comput., Inf. Control, vol. 17, no. 3, 2021, 949-957, 10.24507/ijicic.17.03.949.

[12] J. Abbas, "The 2-Additive Choquet Integral of Bi-capacities". In: L. Rutkowski, R. Scherer, M. Korytkowski, W. Pedrycz, R. Tadeusiewicz and J. M. Zurada (eds.), Artificial Intelligence and
Soft Computing. ICAISC 2019. Lecture Notes in Computer Science, vol. 11508, 2019, 287-295, 10.1007/978-3-030-20912-4_27.

[13] R. I. Sabri, M. N. Mohammedali and J. Abbas, "An Application of Non-additive Measures and Corresponding Integrals in Tourism Management", Baghdad Science Journal, vol. 17, no. 1, 2020, 172-177, 10.21123/bsj.2020.17.1.0172.

[14] M. Hesham and J. Abbas, "Multi-criteria Decision Making on the Best Drug for Rheumatoid Arthritis", Iraqi Journal of Science, vol. 62, no. 5, 2021, 1659-1665, 10.24996/ijs.2021.62.5.28.

[15] J. Abbas, "Shilkret Integral Based on Binary-Element Sets and its Application in The Area of Synthetic Evaluation", Engineering and Technology Journal, vol. 33, no. 3, 2015, 571-577.

[16] J. Abbas, "Logical Twofold Integral”, Engineering and Technology Journal, vol. 28, no. 3, 2010, 477-483.

[17] F. Kareem and J. Abbas, "A Generalization of the Concave Integral in Terms of Decomposition of the Integrated Function for Bipolar Scales", Journal of Applied Sciences and Nanotechnology, vol. 1 , no. 4, 2021, 81-90, 10.53293/ jasn.2021.3985.1065. 